\title{
Preparation and Characterization of UV Curable Complex System Based on Vinyl Resin and Polyurethane Acrylate Prepolymer
}

\author{
ZHAO Can ${ }^{1, a}$, SUN YeNan ${ }^{1, b}$, XIAO JiJun ${ }^{1, c^{*}}$ \\ ${ }^{1}$ College of Material Science and Engineering, Hebei University of Science and Technology, \\ Shijiazhuang 050018, China \\ a1245857242@qq.com,b1907225351@qq.com, cjijunxiao@aliyun.com \\ * Corresponding author XIAO JiJun
}

Keywords: UV-cuable, vinyl resin, polyurethane acrylate, complex system, properties

\begin{abstract}
A UV curable complex system with vinyl resin and polyurethane acrylate photo-polymerization was investigated. The UV curing process of complex system was traced by IR. Thermal decomposition temperature of UV cured film for various system were determined by thermogravimetric analysis(TGA). And physical properties of those curing films were analyzed and compared. The results show The results show the thermal decomposition temperature, and alkali resistance and hardness of the product gradually decreased with the increase in the proportion of polyurethane acrylic prepolymer.The vinyl resin pre polymer were synthesized by epoxy resin E-51 and acrylic acid.The polyurethane pre polymer was prepared by the reaction of polyether -1000 and TDI-80 with hydroxyethyl ester as the end capping agent. The two kinds of products were mixed in different proportions, and the vinyl resin was modified by polyurethane. Finally, diluent and photo initiator were added to the UV curing of products. The results showed that the vinyl resin and polyurethane prepolymer were successfully prepared. The properties of the products were evaluated by IR, TGA and other methods. The alkali resistance and hardness of curing films have been tested.
\end{abstract}

\section{Introduction}

UV curing technology is increasingly demanded in coating industry due to its numerous advantages such as low VOC, high-speed curing in ambient temperature, low energy consumption and its environmentally friendly technique, which have found a great application as protective coating on polycarbonate sheets, optical filters, lenses, displays and compact discs ${ }^{[1]}$. The UV-curable coatings consist of oligomer, active diluent (monomer) and photoinitiator, so the properties of coating film, such as hardness, abrasive resistance, flexibility and weatherability mainly depend on the oligomer structure and its concentration in the formulation ${ }^{[2]}$. In the process of photo-polymerization, the content of the photo initiator would determine the curing degree of the ${ }^{1}$ polymer $^{[3]}$. Besides, the presence of radical scavengers, the reactivity and viscosity of the oligomer, he wavelength and intensity of the UV radiation all could affect the performance of the UV curing film ${ }^{[4]}$. 
During the last few decades, vinyl resin is extensively used as oligomer for UV coatings due to its unique properties including excellent adhesion on substrates, hardness, chemical resistance $^{[5]}$. However, UV-curable vinyl resin coatings are limited in some fields by its poor flexibility and toughness. The polyurethane is used as oligomer for UV coatings due to its outstanding flexibility. So the two kinds of products were mixed in different proportions, and the vinyl resin was modified by polyurethane.

\section{Experiment}

\subsection{Materials}

The raw materials used were as followed: epoxy resin (E-51, Tianjin Xinhua resin plant) and acrylic acid (AA, Tianjin Fuchen chemical reagent factory) and tetraethylammonium bromide (TEAB, Tianjin Kermel Chemical Reagent Co. Ltd), 4-hydroxyanisole (HDNS, Tianjin Tianjiao Chemicals Co. Ltd), polyether diol (DL-1000, Shandong Bluestar Dongda Chemical Co., Ltd), toluene diisocyanates (TDI, Tianjin Bodi Chemical Co., Ltd.), 1-hydroxy-cyclohexyl-phenyl-ketone (Irgacure 184, BASF) and Tripropylene glycol diacrylate (TPGDA) (Tianjin Tianjiao Chemical Co. Ltd). 2-hydroxyethyl acrylate (HEA, Beijing Dongfang Chemical Plant) and quinol (Shanxi coking group company Limited).

\subsection{Preparation of UV Curable Complex System Based on Vinyl Resin and Polyurethane Acrylate Prepolymer}

\subsubsection{Preparation of vinyl resin}

A certain amount of E-51 and TEAB as a catalyst were added into the flask equipped with a reflux condenser, a mechanical stirrer and a thermometer, and heated to complete melting. A certain amount mixture of AA and HDNS as an inhibitor were added into the flask by gradually dropping for $0.5 \mathrm{~h}$ at $90^{\circ} \mathrm{C}$. Then the system was heated to $100 \sim 110^{\circ} \mathrm{C}$ and maintained for $4 \mathrm{hrs}$. Vinyl resin was obtained. The reaction formula of E-51 with AA is shown in Figure1.

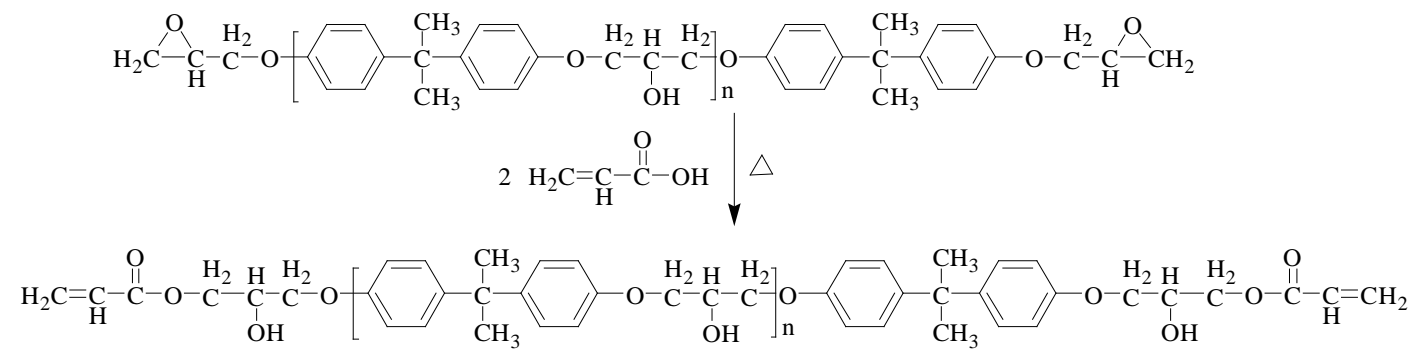

Figure 1 Schematic illustration of the polymerization process for vinyl resin.

\subsubsection{Preparation of polyurethane acrylic resin}

First, a certain amount of DL-1000 was added into the flask equipped with a reflux condenser, a mechanical stirrer and a thermometer, and heated to $70^{\circ} \mathrm{C}$. Then a certain amount of TDI-80 and DBTL were added into the flask, and stirred and maintained for $4 \mathrm{hrs}$ at $80^{\circ} \mathrm{C}$. A certain amount mixture of HEA and quinol were added into the flask, then the system was maintained at $80 \pm 2{ }^{\circ} \mathrm{C}$ for $3 \mathrm{~h}$. Polyurethane acrylic resin was obtained. The reaction formula of DL-1000, TDI with HEA is shown in Figure 2. 


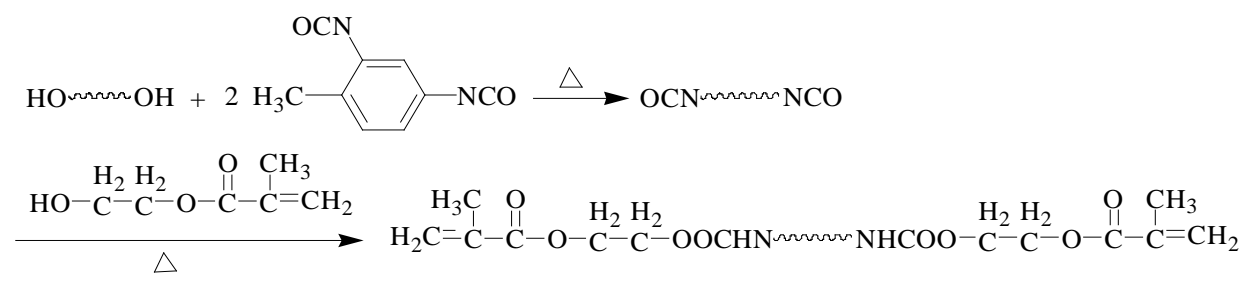

Figure 2 The reaction equation of polyurethane acrylic resin.

\subsubsection{UV-curable formulation}

UV-curable formulations are given in Table 1. High-speed mixing was performed on the formulations for 10 minutes.

Table 1 UV-curable formulations.

\begin{tabular}{|c|c|}
\hline Materials & Weight/g \\
\hline Epoxy resin (E-51) & 57 \\
\hline Traethylammonium bromide (TEAB) & 0.7905 \\
\hline Acrylic acid (AA) & 22.05 \\
\hline 4-Hydroxyanisole (HDNS) & 0.063 \\
\hline Polyether diol (DL-1000) & 150 \\
\hline Toluene diisocyanates (TDI-80) & 52.2 \\
\hline Hydroxyethyl methacrylate (HEMA) & 34.8 \\
\hline Dibutyltin dilaurate (DBTL) & 0.1185 \\
\hline Quinol & 0.04 \\
\hline Tripropylene glycol diacrylate (TPGDA) & 158.531 \\
\hline Photoinitiator 1173 & 9.5119 \\
\hline
\end{tabular}

\subsection{Characterization}

Fourier transform infrared (FTIR) spectra of the products were collected on a Nicolet 6700/Fourier transform-Raman modules (ThermoFisher, Waltham, Massachusetts, USA). The thermal properties of the samples were determined by Sta449C Netzsch (Germany) thermogravimetric analyzer under nitrogen atmosphere, and the temperature was raised to $600{ }^{\circ} \mathrm{C}$ at $10{ }^{\circ} \mathrm{C} / \mathrm{min}$. The alkali resistance and hardness of Curing films were tested according to GB/T 9265-2009 and GB/T 1730-93, respectively.

\section{3 . Results and Discussions}

\subsection{Infrared analysis of UV curing processing}

In order to investigate the UV curing processing of vinyl resin and polyurethane acrylate complex system, the infrared spectra of the system with different irradiated time are shown in Fig.3. The main curing principle of the resin is the radical polymerization of the double band. Therefore, the degree of reaction can be monitored by IR-spectroscopy as the decrease in the initial double bond due to the polymerization. Fig.3 shows that the characteristic absorption peaks of double bonds at $810 \mathrm{~cm}-1$ and $985 \mathrm{~cm}-1$ were apparently weakened with increasing the exposure time. 


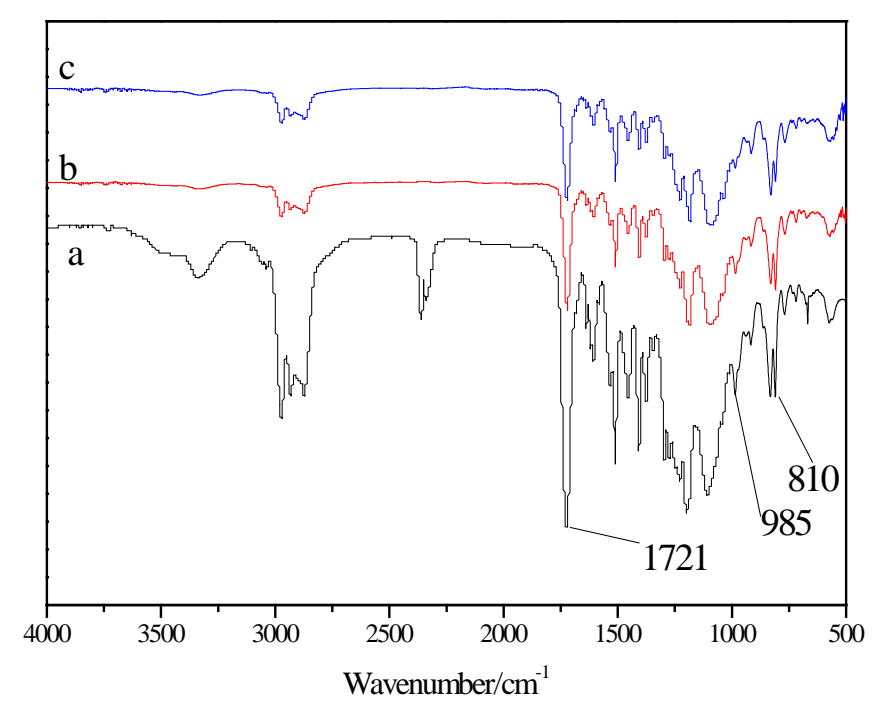

Figure 3 IR spectra of the UV-cured products of the vinyl resin prepolymers irradiated with UV light at different times(a:0s, b:5s, and c: 10s).

\subsection{TGA of UV cured film}

As shown in Figure.4, thermogravimetric analysis (TGA) revealed that the temperature of $10 \%$ weight loss (Td10) for UV cured complex system was about $300^{\circ} \mathrm{C}$. These data indicate that $\mathrm{Td}$ of film depends on the main chain structure of resin. The decomposition temperature of UV cured vinyl resin and polyurethane acrylate complex film was between that of vinyl resin and polyurethane acrylate film.

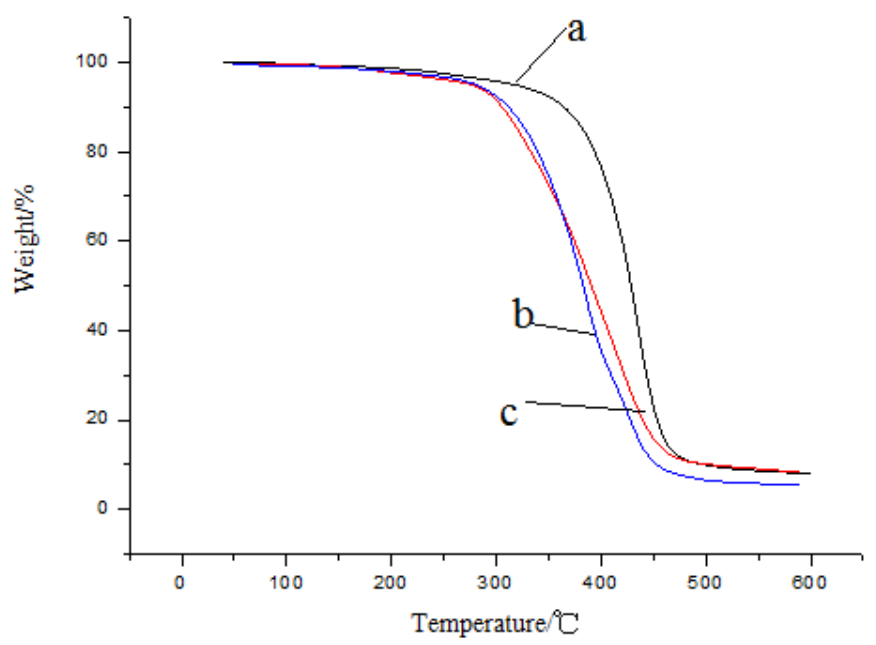

Figure 4 TGA curves of UV cured vinyl resin(a), complex(b) and polyurethane acrylic(c) system.

\subsection{Alkali resistance of UV cured film}

The alkali resistance of cured film for different proportions of polyurethane acrylic and the vinyl resin were measured, and the results are shown in Fig5. With the decrease in the proportion of 
polyurethane acrylic prepolymer, the alkali resistance of cured film gradually increase. This is because that acrylic polyurethanes contain small amounts of polar groups and a lot of ester bonds.

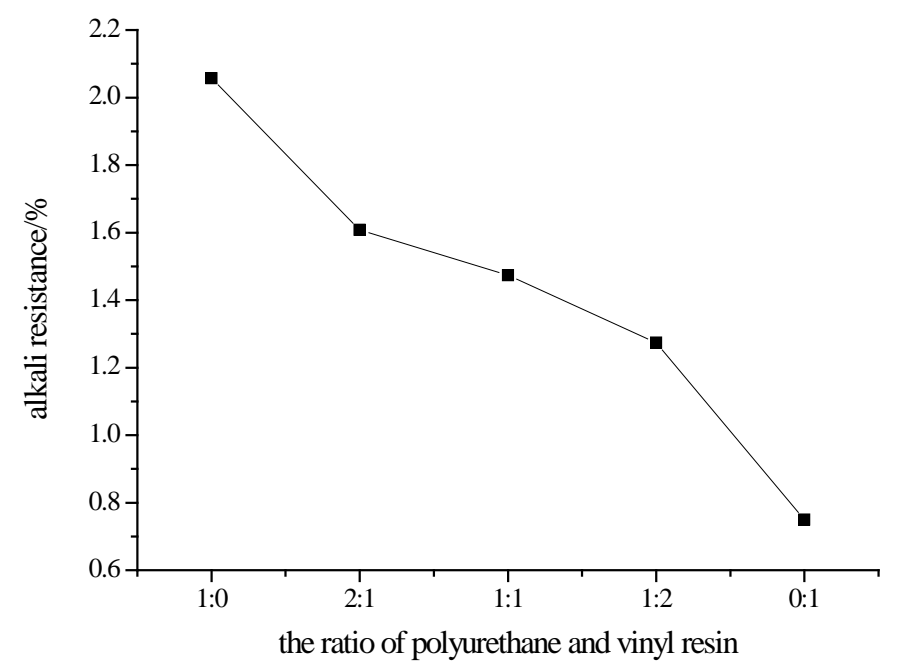

Figure 5 Alkali resistance of UV cured film.

\subsection{Hardness analysis of modified vinyl resin film}

It can be seen from Table 2, with the increase in proportion of polyurethane prepolymer increases, the hardness of the curing film gradually decreased. This is because the curing film contains more secondary hydroxyl, it can be indicating that polyurethane increase the hardness of products.

Table 2 The hardness of modified vinyl resin.

\begin{tabular}{|c|c|}
\hline the ratio of vinyl resin and polyurethane & hardness \\
\hline $1: 0$ & 0.071 \\
\hline $2: 1$ & 0.072 \\
\hline $1: 1$ & 0.078 \\
\hline $1: 2$ & 0.082 \\
\hline $0: 1$ & 0.179 \\
\hline
\end{tabular}

\section{Conclusion}

In this paper, the vinyl resin and polyurethane prepolymer were successfully prepared. IR spectroscopy was used to investigate the curing process for the various UV-curable system. The effects of UV curing time and the amount of polyurethane on the properties of various system were discussed. The results show the thermal decomposition temperature, and alkali resistance and hardness of the product gradually decreased with the increase in the proportion of polyurethane acrylic prepolymer.

\section{Acknowledgment}

The authors would like to thank Hebei Key Laboratory of Material Near-Net Forming Technology for financial support of this research. 


\section{References}

[1] Mehnert R, Pincus A, Janorsky I, Stowe R, Berejka A, editors. UV \& EB curing technology \& equipment. London: SITA Technology Ltd, John Wiley \& Sons; 1998. p. 1-247.

[2] B.K. Kim, Y.H.Cho,J.S. Lee, Polymer 41 (1325) (2000).

[3] X.Y. Xiao, C.C. Hao, Colloids Surf., A 359 (82) (2010).

[4] K. Studer, C.Decker, E. Beck, R. Schwalm, Prog. Org. Coat. 48 (101) (2003).

[5] G. Xu, W.F. Shi, Prog. Org. Coat. 52 (2005) 110-117. 